\title{
Seguridad e inmunogenicidad de la vacuna oral anticólera CVD-103 HgR: estudio en 29 voluntarios
}

\begin{abstract}
Elizabeth Castañeda', Magaly Chinchilla', Martha Velandia², Fernando de la $\mathrm{Hoz}^{2}$
Resumen

Con el fin de determinar si la vacuna CVD-103 HgR era segura e inmunogénica, se administró una dosis a 29 voluntarios sanos, en el Laboratorio de Microbiología del Instituto Nacional de Salud. Se anotaron los efectos secundarios durante siete días después de la ingestión de la vacuna. Se tomaron 5 muestras de sangre los días 0,7 , 10,14 y 28 después de la toma de la dosis. Se determinaron los títulos de anticuerpos antibacterianos por medio de la técnica vibriocida y anticuerpos antitoxina IgG e IgA por medio de la técnica de ELISA. Nueve voluntarios (31\%) manifestaron efectos secundarios leves (náuseas, dolor abdominal o cefalea) los cuales fueron más frecuentes durante el primero y segundo días después de haber tomado la vacuna. El mayor porcentaje de seroconversión en anticuerpos vibriocidas fue de 93\% a los 7 días, en anticuerpos antitoxina IgG fue de $71,4 \%$ a los 14 días y para antitoxina IgA en igual porcentaje a los 10 días. La mayor media geométrica de anticuerpos vibriocidas fue de 1.538 (1.0642.223) a los 10 días, la de anticuerpos antitoxina IgG fue de 251 (212-297) a los 14 días y para anticuerpos antitoxina IgA fue de 48 (40-57) a los 10 días. Se concluyó que la vacuna era segura e inmunogénica en esta población.
\end{abstract}

\begin{abstract}
Summary
The safety and immunogenicity of a single dose of CVD-103 HgR oral vaccine was evaluated on 29 healthy volunteers from the Instituto Nacional de Salud. Side effects were registered during the 7 days following the ingestion of the dose. Blood was taken on the first day and on days 7, 10,14 and 28. Antibacterial antibody titers were determined using vibriocidal techniques and antitoxin antibodies (classes $\lg G$ and $\lg A$ ) using the ELISA test. Nine volunteers presented slight side effects (nausea, abdominal pain and cephalea) which were more frequent on the first and second day after vaccination. The highest percentage of seroconversion (93\%) of antibacterial antibodies using vibriocidal techniques was obtained 7 days after the administration of the dose. Seroconversion for antitoxin antibodies, IgG and IgA, was obtained in $71.4 \%$ of the participants on the fourteenth and tenth day, respectively. A single dose of the oral vaccine CVD-103HgR was found to be safe and immunogenic.
\end{abstract}

Al llegar el cólera a Colombia, se tomaron las medidas recomendadas por la OPS/OMS para controlar la epidemia las cuales incluyeron el establecimiento de sistemas de vigilancia de la enfermedad, el diagnóstico bacteriológico, la

Grupo de Microbiología, INS.

2 Grupo de Vigilancia Epidemiológica, INS. organización de programas eficaces de tratamiento para prevenir la mortalidad, la promoción de la educación para la salud y otras relacionadas con el abastecimiento de agua y alimentos no contaminados $(1,2)$. En esos momentos, la OPS/ OMS volcó nuevamente la atención sobre el desarrollo de una vacuna eficaz contra el cólera, la cual podría ser una medida que complementaría las actividades anteriormente descritas (3); 
por tanto, convocó a un comité de expertos el cual recomendó realizar en América Latina estudios de seguridad e inmunogenicidad (estudios de fase II) con las nuevas vacunas orales: la de células enteras muertas más la subunidad $\mathrm{B}$ recombinante de la toxina ( $\mathrm{CE} / \mathrm{sBr}$ ) y la vacuna de células vivas CVD-103 HgR (3).

La vacuna CVD-103 HgR es una cepa de Vibrio cholerae 01 subtipo Inaba, biotipo clásico, a la cual se le ha inducido una deleción en el gen que codifica para la subunidad A de la toxina permaneciendo intacto el gen de la subunidad B. Esta cepa coloniza el intestino eficientemente, no produce reacciones adversas y contiene un marcador genético que la diferencia de la cepa silvestre. Adicionalmente, induce tanto inmunidad antibacteriana como inmunidad antitoxina (3-6). En estudios de fase II se ha descrito la cinética de la respuesta de anticuerpos vibriocidas observándose el mayor título hacia el día 10 (7) y actualmente se está realizando un estudio de eficacia (fase III) en Jakarta, Indonesia, que ha involucrado aproximadamente a 67.000 personas en riesgo de infección por cólera (8).

Para conocer el comportamiento de esta vacuna en nuestro medio, se decidió determinar la seguridad y medir la estimulación de la respuesta. inmune humoral en un grupo de voluntarios sanos.

\section{Materiales y métodos}

Voluntarios: participaron en el estudio 29 personas sanas, en edades comprendidas entre los 20 y 40 años, los cuales firmaron un consentimiento.

Vacuna: se utilizó la vacuna oral CVD-103 HgR que consiste en $5 \times 10^{9}$ UFC de la cepa $569 \mathrm{~B}$ de Vibrio cholerae 01 subtipo Inaba, biotipo clásico, la cual tiene una deleción en el gen que codifica para la subunidad $A$ de la toxina, permaneciendo intacto el gen de la subunidad $B$ y adicionalmente posee un gen que codifica resistencia $\mathrm{al}_{\mathrm{Hg}}{ }^{2+}$.

La presentación de la vacuna (Orochol BernaInstituto Suizo de Sueroterapia y Vacunación Berna) consiste en dos sobres, uno de los cuales contiene el buffer que neutraliza el ácido estomacal y está compuesto por $2,5 \mathrm{~g}$ de $\mathrm{NaHCO}_{3}$ y $1,65 \mathrm{~g}$ de ácido ascórbico, el otro sobre contiene $5 \times 10^{9}$ organismos vacunales liofilizados y $25 \mathrm{mg}$ de aspartame.

Administración: el sobre con el bufferse disolvió en $100 \mathrm{~mL}$ de agua destilada y se le adicionó el sobre con la bacteria liofilizada; esta mezcla se administró inmediatamente a cada uno de los voluntarios, a quienes se les solicitó no ingerir alimentos por un período de 90 minutos antes y después de recibir la vacuna.

Vigilancia de efectos secundarios: se repartió a cada voluntario un formulario que indagaba la presencia de fiebre, cefalea, malestar, náuseas, vómito, dolor abdominal y diarrea (tres o más deposiciones blandas o líquidas en 24 horas). Todos los síntomas fueron vigilados durante los siete primeros días después de la ingestión de la dosis.

Serología: se recolectaron $5 \mathrm{~mL}$ de sangre en los días $0,7,10,14$ y 28 después de ingerida la vacuna. En el suero se determinó la presencia de anticuerpos antibacterianos por medio de la técnica vibriocida; se consideró como seroconversión un incremento igual o mayor a dos diluciones con respecto a la muestra del día 0 (9). Los anticuerpos antitoxina IgG e IgA se determinaron por medio de la técnica de ELISA; se consideró como seroconversión un incremento igual o mayor a 1,5 veces con relación al título del día 0 (10).

Métodos estadísticos: se obtuvo la media geométrica de los títulos de todas las muestras. Los promedios obtenidos en cada muestra se compararon usando la prueba t de Student para muestras apareadas; el nivel de confianza usado para las comparaciones fue del 95\% (11).

\section{Resultados}

Efectos secundarios: los 29 voluntarios llenaron el formulario de vigilancia de efectos secundarios. El día de la ingesta de la vacuna, dos voluntarios presentaron dos síntomas y 7 voluntarios sólo uno, distribuidos así: 4 presentaron náuseas, 3 dolor abdominal, 3 cefalea y 1 malestar abdominal. La presencia de efectos secundarios disminuyó en los días siguientes. La intensidad de los síntomas fue leve y ninguno de los 
pacientes requirió atención médica o cuidados especiales.

Respuesta inmune: se tomaron 143 muestras de sangre distribuidas así: 29 los días 0,7 y 28 y 28 muestras los días 10 y 14 . El mayor porcentaje de seroconversión (93\%) para los títulos vibriocidas se obtuvo el séptimo día. Las diferencias entre los porcentajes de seroconversión de las muestras tomadas el día 0 y las siguientes cuatro muestras fueron estadísticamente significativas (tabla 1). La mayor media geométrica de los títulos vibriocidas fue de 1.538 en el decimo día con un rango entre 1.064-2.223, después descendió hasta 288 en el día 28 (figura 1).

Tabla 1. Porcentajes de seroconversión de anticuerpos vibriocidas y antitoxina en los voluntarios que ingirieron la vacuna CVD $103 \mathrm{Hg} \mathrm{R}$, con relación al día 0.

\begin{tabular}{lllll}
\hline & \multicolumn{4}{c}{ DIAS } \\
\cline { 2 - 5 } PRUEBAS & $\mathbf{7}$ & $\mathbf{1 0}$ & $\mathbf{1 4}$ & $\mathbf{2 8}$ \\
\hline VC & 93 & 92 & 92 & 86 \\
AT IgG & 27,6 & 60,7 & 71,4 & 62,1 \\
AT IgA & 37,9 & 71,4 & 67,8 & 41,4 \\
\hline n & 29 & 28 & 28 & 29 \\
\hline
\end{tabular}

VC: vibriocida

AT IgG: antitoxina $\lg G$

AT IgA: antitoxina IgA

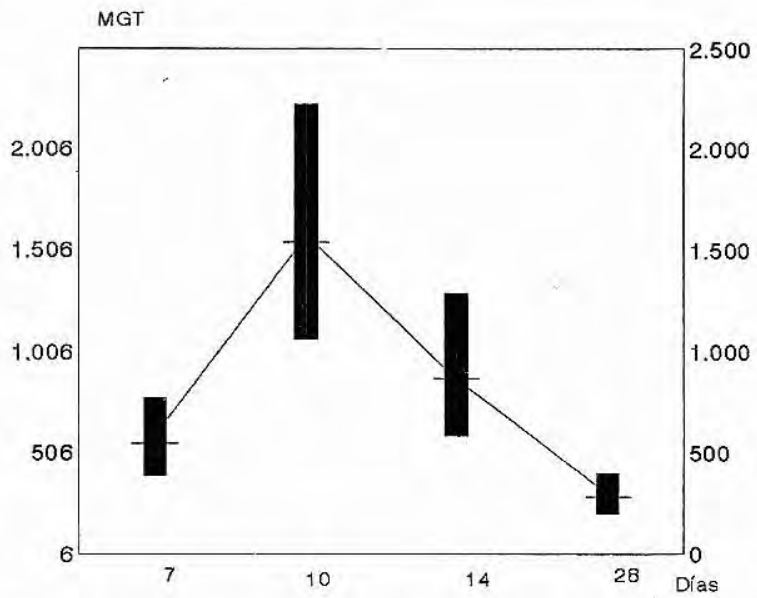

Figura 1. Medias geométricas de los títulos de anticuerpos vibriocidas en los diferentes días.
Con relación a los anticuerpos antitoxina de clases $\lg \mathrm{G}$ e $\lg \mathrm{A}$, el porcentaje de seroconversión más alto fue de $71,4 \%$ en los días 14 y 10 , respectivamente (tabla 1). La media geométrica de los títulos IgG antitoxina fue aumentando paulatinamente hasta llegar en el día 14 a 251 con un rango entre $212-297$, disminuyendo discretamente en la última medición (figura 2); la máxima media geométrica para la lgA antitoxina fue de 48 (rango 40-57) y fue obtenida el día 10, descendiendo en las observaciones de los días 14 y 28 con un decremento más acentuado que el de la lgG (figura 3).

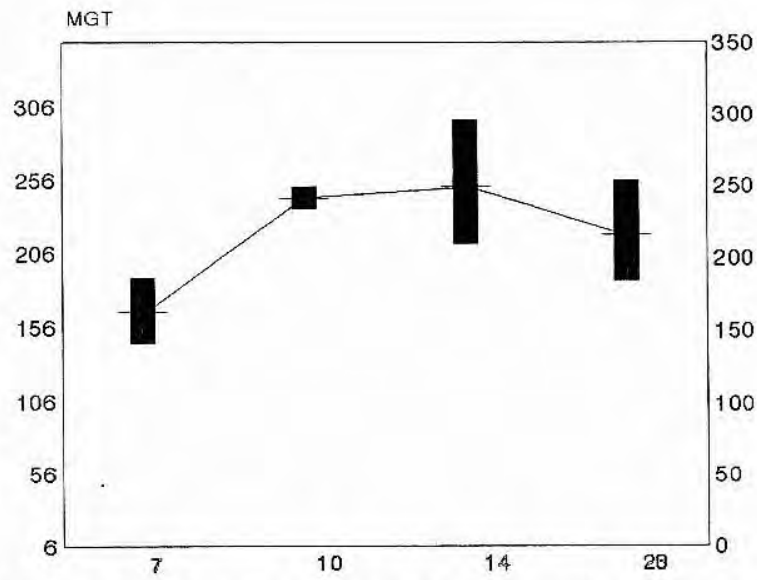

Figura 2. Medias geométricas de los títulos de lgG antitoxina en los diferentes días.

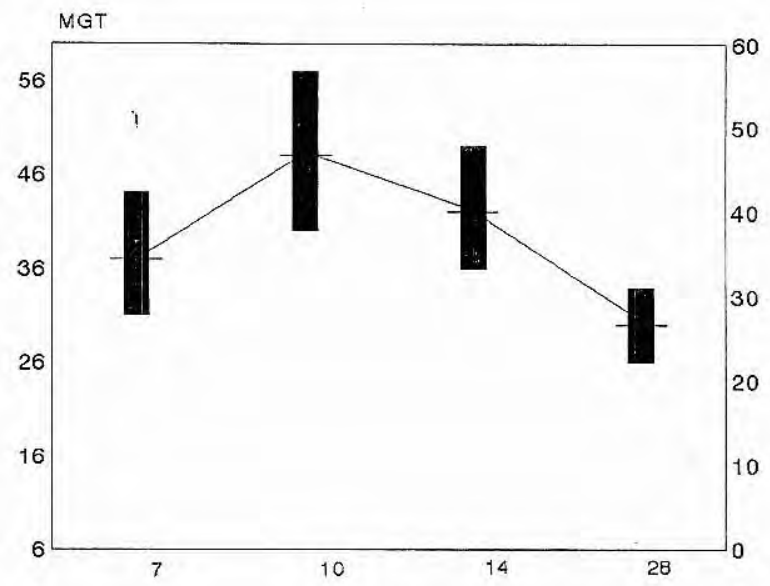

Figura 3. Medias geométricas de los títulos de IgA antitoxina en los diferentes días. 


\section{Discusión}

Se han llevado a cabo varias evaluaciones de la seguridad de la posible vacuna oral contra el cólera denominada CVD $103 \mathrm{HgR}$, en las cuales se ha demostrado que ésta es bien tolerada tanto en voluntarios norteamericanos (4) como en adultos tailandeses (5) donde el $11 \%$ y el $2,5 \%$, respectivamente, dijeron haber padecido algún síntoma colateral, siendo éstos clasificados como leves, lo que es comparable con los hallazgos de este ensayo.

La vacuna compuesta por $V$. cholerae vivo atenuado produce una respuesta inmune similar a la originada por la infección natural, gracias a su capacidad de colonizar el intestino delgado proximal, estimulando tanto la inmunidad antitoxina como antibacteriana; se ha asociado esta última respuesta con una alta protección contra el cólera (12).

La tasa de seroconversión de anticuerpos vibriocidas obtenida en este estudio es comparable a las de estudios previos hechos en áreas no endémicas como Estados Unidos y Suecia, en los cuales se han obtenido porcentajes del 88 y $92 \%$, respectivamente $(4,6)$; también se han informado variaciones del $22 \%$ al $63 \%$ en civiles y soldados tailandeses (13). El no pertenecer los voluntarios a un área endémica, se demostró en la baja media geómetrica de los títulos de anticuerpos vibriocidas obtenida en la muestra inicial y que ascendió posteriormente en el día 10 , correlacionándose con las obtenidas en los estudios de voluntarios norteamericanos, suecos y civiles tailandeses $(4,6,13)$. Llama la atención el hecho que estos títulos disminuyan tan rápidamente (28 días), sugiriendo la necesidad de establecer posibles resfuerzos (14).

La magnitud de la respuesta de seroconversión de los anticuerpos antitoxina lgG $(71,4 \%)$ es comparable con la de los estudios llevados a cabo en adultos sanos tailandeses $(75 \%)$ y en adultos suecos (76\%); en contraste, es mucho mayor que la encontrada en soldados tailandeses (22-27\%). En este último estudio, se observó el papel que juega, en el desarrollo de la inmunidad las características del huésped y el contacto previo con $V$. cholerae, ya que en voluntarios sanos provenientes de zonas rurales y de estratos socieconómicos bajos, con exposición previa a la infección, se presentaron menores tasas de seroconversión de anticuerpos antitoxina y antivibriocida que en los voluntarios que pertenecían a zonas urbanas y a estratos socieconómicos medios (13); estos últimos son similares a nuestros voluntarios encontrándose también similaridad en los resultados.

El porcentaje de seroconversión de los títulos de anticuerpos antitoxina IgA fue del $71,4 \%$, lo cual es reflejo de la respuesta presentada en el lumen intestinal, donde la lgA secretora provee una protección específica a la mucosa evitando la unión de la toxina a las células epiteliales. La duración de esta respuesta es corta, de una semana a dos meses, pero queda una memoria inmunológica que puede ser reestimulada en una segunda exposición proporcionando una rápida y eficiente respuesta (15).

Se corroboran los hallazgos de estudios previos donde se evidenció que una única dosis de CVD 103-HgR es segura e inmunogénica en individuos con un bajo nivel previo de anticuerpos anticólera (13).

El próximo paso debe ser la realización de ensayos de eficacia para evaluar la protección inducida por esta posible vacuna oral contra el cólera, aunque en este momento y, afortunadamente en Colombia, la enfermedad ha disminuido verticalmente por lo cual no se lograría obtener el número necesario de casos para evaluar la eficacia de las posibles vacunas orales.

\section{Agradecimientos}

A los voluntarios por su gran colaboración durante el desarrollo del estudio. Al doctor William Rojas de la Corporación para Investigaciones Biológicas (CIB) de Medellín por establecernos el contacto con el Centro para Desarrollo de Vacunas (CVD) de Baltimore y a los doctores Fernando Noriega y Myron Levine del CVD por el envío de las dosis de vacuna requeridas para el estudio.

\section{Referencias}

1. Organización Panamericana de la Salud. La situación del cólera en las Américas. Boletín epidemiológico 1991;12:1-24. 
2. World Health Organization - Centers for Disease Control. Guidelines for cholera control, 1986.

3. Organización Panamericana de la Salud. Evaluación de las vacunas contra el cólera. Boletín epidemiológico 1991;12:11-4.

4. Levine MM, Herrington D, Losonsky G, et al. Safety, immunogenicity and efficacy of recombinant live oral cholera vaccines, CVD 103 and CVD $103 \mathrm{HgR}$. Lancet 1988;II:467-70.

5. Migasena S, Pitisuttitham P, Prayurahong B, et al. Preliminary assessment of the safety and immunogenicity of live oral cholera vaccine strain CVD $103 \mathrm{HgR}$ in healthy Thai adults. Infec Immun 1989;57:3261-4.

6. Cryz SJ, Levine MM, Kaper JB, et al. Randomized double-blind placebo controlled trial to evaluate the safety and immunogenicity of the live oral cholera vaccine strain CVD $103 \mathrm{HgR}$ in Swiss adults. Vaccine 1990;8:577-80.

7. Wasserman SS, Losonsky GA, Noriega F, et al. Kinetics of the vibriocidal antibody response to live oral cholera vaccines. Vaccine 1994;12:1000-3.

8. Sack RB, Albert MJ. Cholera vaccine workshop. J Infect Dis 1994; 170:256.

9. Benenson AS, Saad M, Moseiy WH. Serological studies in cholera. 2 . The vibriocidal antibody response of chol- era patients determined by microtechnique. Bull WHO 1968;38:277-85.

10. Holmgren J, Svennerholm AM. Enzyme-linked immunoabsorbent assays for cholera serology. Infect Immun 1973;7:753.

11. Paul E, White F. Seroepidemiology. New York: Oxford Press, 1954.

12. Holmgren J, Clemens J, Sack D, Svennerholm AM. New cholera vaccines. Vaccine 1989;7:94-96.

13. Su-Arehawaratana $P$, Singharaj $P$, Taylor $D$, et al. Studies on the safety and immunogenicity of different immunization regimens of CVD 103- $\mathrm{HgR}$ live oral cholera vaccine in soldiers and civilians in Thailand. $\mathrm{J}$ Infect Dis 1992;165:1042-8.

14. Levine MM, Tacket CO. Recombinant live cholera vaccines. In: Wachsmuth IK, Blake PA, Olsvik O, editors. Vibrio cholerae and cholera. Washington, D.C.: American Society for Microbiology, 1994:395-413.

15. Svennerholm AM, Jonson G, Holmgren J. Immunity to Vibrio cholerae infection. In: Wachsmuth IK, Blake PA, Olsvik O, editors. Vibrio cholerae and cholera. Washington, D.C.:American Society for Microbiology, 1994:257-71. 